\title{
Internal Medicine
}

REVIEW ARTICLE

\section{Elderly Involvement in the Era of COVID-19 Infection}

\author{
Maha Hossam Eldin Ibrahim ${ }^{1}$ and Abeer Awad Abdellatif ${ }^{*}$ \\ ${ }^{1}$ Professor, Internal Medicine and Geriatric Unit, Kasr Al-Aini Hospitals, Cairo University, Egypt \\ ${ }^{2}$ Lecturer, Internal Medicine and Geriatric Unit, Kasr Al-Ainy Hospitals, Cairo University, Egypt
}

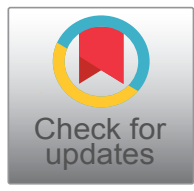

*Corresponding author: Abeer Awad Abdellatif, Lecturer, Internal Medicine and Geriatric Unit, Kasr Al-Ainy Hospitals, Cairo University, PO: 11451, Kasr Al-Aini Street, Cairo, Egypt, Tel: 01002776494

\begin{abstract}
Nowadays, the entire world is facing the outbreak pandemic of COVID-19 infection which is a lethal infection to some extent. The most commonly affected group of population is the elderly due to impaired immunity and multiple co-morbidities they suffer from. From this point of view; we will discuss some important issues about the elderly involvement during COVID-19 pandemic hoping to raise the awareness toward that frail sector of population. Indeed many researches for COVID infection whether regarding outcomes, predictive diagnostics, and management strategies including optimal approach in older people is largely needed.
\end{abstract}

\section{Keywords}

Elderly, COVID-19

\section{Introduction}

In December 2019 outbreak of novel coronavirus infection (COVID-19) started in Wuhan, China rapidly spreading to involve most of the countries worldwide with high mortality rate [1]. COVID-19 is a coronavirus infection, that caused by severe acute respiratory syndrome coronavirus 2 (SARS-CoV-2) which share some similarity with other viral infections as in Severe Acute Respiratory Syndrome (SARS in 2003) caused by SARSCOV-2, Middle Eastern Respiratory Syndrome (MERS in 2013) caused by MERS-CoV and human coronavirus-EMC. Both innate and adaptive immunity are involved in COVID-19 infection [2].

The elderly is most susceptible to infection than the general population with a higher risk for increased mortality rate. These may be due to primary or secondary structural and functional changes of bone marrow, thymus, lymphoid organs, and immune cells associated with aging [3].
Indeed to the physiological changes associated with aging, the elderly become of low capability to fight infection with increasing the risk of autoimmunity, and constitutive low-grade inflammation [3].

Beside the physiological changes occurring with aging, it was reported that the older man is prone to infectious diseases more than an older woman with high pro-inflammatory immune responses and low adaptive immune responses $[4,5]$.

In a lot of studies about aging, there was a link founded between age, diet, nutrients, and immunity in the elderly [6]; the clinical or subclinical micronutrient deficiency is common in older adults; conforming to this fact they may have a role in many age related diseases and decreased immune functions $[7,8]$.

\section{Elderly Affection in COVID-19 Pandemic}

Owing to impaired immunity and multiple co morbidities that the elderly people suffer; as respiratory diseases, cardiovascular disease and diabetes mellitus so they present the most vulnerable group of people susceptible to severe COVID-19 infection with a higher mortality rate in the clinical settings [9-11].

As reported in the early statistical analysis of data in China, patients aged 60-years-old represent higher case-fatality rate (CRF); they represent $81 \%$ of the total deaths from COVID-19 confirmed cases [12]. In a study of 1099 patients with COVID-19 infection in China, they found that patients aged 60 -years-old and above represented $15.1 \%, 27 \%$ of them suffered from severe illness [13]. Although in another study analyzing 4021 COVID-19 confirmed cases, patients aged 60-years-old or more represented $26.2 \%$ of total number of cases

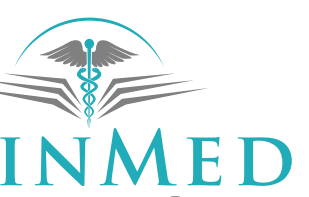

INTERNATIONAL LIBRARY

Citation: Ibrahim MHE, Abdellatif AA (2020) Elderly Involvement in the Era of COVID-19 Infection. Int Arch Intern Med 4:028. doi.org/10.23937/2643-4466/1710028

Received: June 22, 2020: Accepted: September 12, 2020: Published: September 14, 2020

Copyright: (c) $2020 \mathrm{Ibrahim} \mathrm{MHE,} \mathrm{et} \mathrm{al.} \mathrm{This} \mathrm{is} \mathrm{an} \mathrm{open-access} \mathrm{article} \mathrm{distributed} \mathrm{under} \mathrm{the} \mathrm{terms} \mathrm{of} \mathrm{the}$ Creative Commons Attribution License, which permits unrestricted use, distribution, and reproduction in any medium, provided the original author and source are credited. 
with higher rate of mortality which was $5.3 \%$ in comparison to $1.4 \%$ for younger age patients [14].

Several reports during COVID-19 outbreaks in china showed that older age can be a predictor of severity and mortality during COVID-19 infections [15]. Upon a retrospective analysis of data from China and elsewhere they concluded that there is a powerful correlation between age gradient, infection fatality ratio (IFR) and case fatality ratio (CFR) [16]. In March 2020, elderly was the most common affected sector in French with high rate of mortality, they accounted for $20 \%$ of the confirmed cases and $79 \%$ of the total deaths [17].

\section{Clinical Features of COVID-19 Infection in Elderly}

Fortunately the majority of COVID-19 cases are mild with small number of cases require hospitalization and oxygen support (around 14\%), and 5\% only require admission to an intensive care unit [18].

In severe cases of COVID-19; acute respiratory distress syndrome (ARDS), sepsis and septic shock, multiorgan failure are the main causes of mortality [19].

As the elderly people usually present with atypical clinical presentations so there will be a great challenge for their diagnosis and management with great incidence of delayed diagnosis or misinterpretation especially in cases of cognitive impairment together with higher risk for spreading infection during COVID-19 outbreaks and high mortality rate [20]. In many studies upon analysis of clinical characteristics of COVID-19 infection in elderly, it was found that the most obvious symptoms at admission were fever, cough, dyspnea and fatigue which are consistent also with any viral infection. Obviously, dyspnea was the most frequently acquired symptom in dead patients [21].

\section{Outcome of COVID-19 infection in Elderly}

Out of 339 COVID-19 infected patients aged 60-yearsold or more, over $70 \%$ were found suffering from severe infection, higher rate of progression and high case fatality rate (19\%). There were several risk factors associated with poor outcomes in elderly which was a symptom as dyspnea, presence of multiple comorbidities as cardiovascular disease and chronic obstructive pulmonary disease (COPD), or complications as acute respiratory distress syndrome (ARDS). Unfortunately very few numbers of elderly patients that were mechanically ventilated with ARDS survive, therefore with occurrence of ARDS, the incidence of mortality increases dramatically; and so we can suggest ARDS as a predictor for poor outcome [22].

Upon a retrospective analysis study of 82 COVID-19 deaths in Wuhan, China; more than half of dead patients were older than 60 years represented $80.5 \%$ of total deaths with median age 72.5 years. Rate of deaths were more in male (65.9\%). The majority of dead patients had comorbidity $(76.8 \%)$, as hypertension in $56.1 \%$, heart disease in $20.7 \%$, diabetes in $18.3 \%$, cerebrovascular disease in $12.2 \%$, and cancer in $7.3 \%$ [23].

Because the high prevalence of malnutrition in elderly and its impaction in immune status therefore the nutritional assessment and proper management should be essential to determine the risk of infection, the illness course, and the outcome of COVID-19 in older adults [24].

\section{Prevention of COVID-19 Infection in Elderly}

Elderly have the same rights as general population to receive high-quality health care, including intensive care. In older people with probable or suspected COVID-19 it is essential to provide person-centered assessment including understanding the person's life, values, priorities, and preferences for health management. It is essential to ensure multidisciplinary collaboration among physicians, nurses, pharmacists, and other health care professionals to address multimorbidity and functional decline together with caregivers and family members in decision-making and goal-setting throughout the management of older COVID-19 patients [25].

As regarding to protection of elderly people during COVID-19 outbreak, strict instructions should be taken including strict infection control measures, self-quarantine that was established in many countries, it is also important to maintain healthy lifestyle with good nutrition, physical activity and stopping tobacco and alcohol; however this social isolation and loneliness have a bad impact on elderly people with increasing the incidence of cognitive disorders which may lead to further delay in the diagnosis so there must be a good way for better communication between the family, caregivers and medical staff [26]. In attempt for caring of elderly people, the Association for Geriatric Palliative Medicine (FGPG) established a palliative care approach in both inpatient setting and at home [20].

The macro-, micronutrients, and phytonutrients in diet provide a good method for promotion of immune system especially the antioxidants and the anti-inflammatory nutrients that are present in many compounds such as beta-carotene, vitamin C, vitamin E, and polyphenolic compounds $[27,28]$. Also the nutritional status of elderly affects the risk of SARS-CoV-II infection, the clinical course, and the outcomes of COVID-19. And so, the maintenance of host macro- and micronutrient status either by foods, nutrients, or medicines could be an important preventive measure for COVID-19 [29-31].

It is essential to avoid the inappropriate medication prescriptions in patients treated for COVID-19 in order to prevent the undesirable adverse drug events and drug interactions [24].

\section{Conclusion}

In conclusion; owing to vulnerability and multiple co 
morbidities in elderly people, they are the most commonly affected sector with COVID-19 infection with high risk of progression to severe infection and higher rate of mortality, so strict measurements should be taken for protection, close monitoring, early treatment which could be a mean to improve the outcome in the affected elderly patients. Nutritional assessment and management should be an integrated part in prevention of COVID-19 infection in elderly. We are still in great need of more studies to clarify the natural history of disease with serial biological sampling especially in elderly. Currently many researches for COVID infection whether regarding outcomes, predictive diagnostics, and management strategies including optimal approach in older people is largely needed.

\section{Acknowledgements}

We would like to acknowledge our great Kasr Al Ainy Hospital, and its workers, nurses and staff members, for all the support and help in this study and throughout our careers.

\section{Declaration of Interest}

The authors report no conflicts of interest. The authors alone are responsible for the content and writing of the paper.

\section{Funding}

Authors received no funding for this study.

\section{References}

1. Wei-jie Guan, Zheng-yi Ni, Yu Hu, Wen-hua Liang, Chunquan Ou, et al. (2020) Clinical characteristics of coronavirus disease 2019 in China. N Engl J Med 382: 1708-1720.

2. Hofman MR, van den Hanenberg F, Sierevelt IN, Tulner CR (2017) Elderly patients with an atypical presentation of illness in the emergency department. Neth J Med 75: 241246.

3. Sadighi Akha AA (2018) Aging and the immune system: An overview, J Immunol Methods 463: 21-26.

4. Márquez EJ, Chung $\mathrm{CH}$, Marches R, Rossi RJ, Nehar-Belaid D, et al. (2020) Sexual-dimorphism in human immune system aging. Nature Communications 11: 751.

5. Jaillon S, Berthenet K, Garlanda C (2019) Sexual dimorphism in innate immunity. Clin Rev Allergy Immunol 56: 308-321.

6. Yang Y, Li W, Zhang Q, Zhang L, Cheung T, et al. (2020) Mental health services for older adults in China during the COVID-19 outbreak. Lancet Psychiatry 7: 19.

7. Hoffman R (2017) Micronutrient deficiencies in the elderly-could ready meals be part of the solution? J Nutr Sci 6: 2 .

8. Conzade R, Koenig W, Heier M, Schneider A, Grill E, et al. (2017) Prevalence and predictors of subclinical micronutrient deficiency in german older adults: Results from the population-based kora-age study. Nutrients 9: 1276.

9. Chen N, Zhou M, Dong X, Qu J, Gong F, et al. (2020) Epidemiological and clinical characteristics of 99 cases of 2019 novel coronavirus pneumonia in Wuhan, China: A descriptive study. Lancet 395: 507-513.
10. Onder G, Rezza G, Brusaferro S (2020) Case-fatality rate and characteristics of patients dying in relation to COVID-19 in Italy. JAMA.

11. Weiss P, Murdoch DR (2020) Clinical course and mortality risk of severe COVID-19. Lancet 395: 1014-1015.

12. Lang Wang, Wenbo He, Xiaomei Yu, Dalong Hu, Mingwei Bao, et al. (2020) Coronavirus disease 2019 in elderly patients: Characteristics and prognostic factors based on 4-week follow-up. J Infect 80: 639-645.

13. Liu Y, Gayle AA, Wilder-Smith A, Rocklov J (2020) The reproductive number of COVID-19 is higher compared to SARS coronavirus. J Travel Med 27.

14. (2020) COVID-19: Point épidémiologique du 15 mars 2020. Santé Publique France.

15. Ziggi Ivan Santini, Paul E Jose, Erin York Cornwell, Ai Koyanagi, Line Nielsen, et al. (2020) Social disconnectedness, perceived isolation, and symptoms of depression and anxiety among older Americans (NSHAP): A longitudinal mediation analysis. Lancet Public Health 5: 62-70.

16. Piercarlo Sarzi-Puttini, Valeria Giorgi, Silvia Sirotti, Daniela Marotto, Sandro Ardizzone, et al. (2020) COVID-19, cytokines and immunosuppression: What can we learn from severe acute respiratory syndrome? Clin Exp Rheumatol 38: $337-342$.

17. Swiss Academy of Medical Sciences (2020) COVID-19 pandemic: Triage for intensive care treatment under resource scarcity.

18. (2020) Vital surveillances: The epidemiological characteristics of an outbreak of 2019 novel coronavirus diseases (COVID-19)-China, 2020. China CDC Weekly 2: 113-122.

19. Yang $X, Y u Y, X u$ J, Shu H, Xia J, et al. (2020) Clinical course and outcomes of critically ill patients with SARSCoV-2 pneumonia in Wuhan, China: A single-centered, retrospective, observational study. Lancet Respir Med 8: 475-481.

20. (2020) The epidemiological characteristics of an outbreak of 2019 novel coronavirus diseases (COVID-19) in China. Zhonghua Liu Xing Bing Xue Za Zhi 41: 145-151.

21. Verity R, Okell LC, Dorigatti I, Winskill $P$, Whittaker $C$, et al. (2020) Estimates of the severity of coronavirus disease 2019: A model-based analysis. Lancet Infect Dis 20: 669-677.

22. Wu Z, McGoogan JM (2020) Characteristics of and important lessons from the coronavirus disease 2019 (COVID-19) outbreak in China: Summary of a report of 72314 cases from the Chinese Center for Disease Control and Prevention. JAMA.

23. Bicheng Zhang, Xiaoyang Zhou, Yanru Qiu, Fan Feng, Jia Feng, et al. (2020) Clinical characteristics of 82 death cases with COVID-19. Med Rxiv.

24. Lipsitch M, Swerdlow DL, Finelli L (2020) Defining the epidemiology of covid-19-studies needed. N Engl J Med 382: 1194-1196.

25. World Health Organization (2020) Clinical management of severe acute respiratory infection (SARI)' when COVID-19 disease is suspected: Interim guidance.

26. Yang Y, Lu QB, Liu MJ, Yi-Xing Wang, An-Ran Zhang, et al. (2020) Epidemiological and clinical features of the 2019 novel coronavirus outbreak in China. Med Rxiv.

27. Zhang L, Liu Y (2020) Potential interventions for novel coronavirus in China: A systematic review. J Med Virol 92: 479490. 
28. Calder PC, Carr AC, Gombart AF, Eggersdorfer M (2020) Optimal nutritional status for a well functioning immune system is an important factor to protect against viral infections. Nutrients 12: 1181.

29. Kritas SG, Ronconi G, Caraffa Al, Gallenga CE, Ross R, et al. (2020) Mast cells contribute to coronavirus-induced inflammation: New anti-inflammatory strategy, J Biol Regul Homeost Agents 34: 9-14.
30. Conti P, Ronconi G, Caraffa A, Gallenga CE, Ross R, et al. (2020) Induction of pro-inflammatory cytokines (IL-1 and IL-6) and lung inflammation by coronavirus-19 (COVI-19 or SARS-CoV-2): Anti-inflammatory strategies. J Biol Regul Homeost Agents 34: 327-331.

31. Maggini S, Pierre P, Calder PC (2018) Immune function and micronutrient requirements change over the life course. Nutrients 10: 1531. 